\title{
Electromagnetic Waves Propagation Modes of Hot Plasma Located in Rotating Electromagnetic Field
}

\author{
Richard Mwamba Mutombo, Abert Kazadi Mukenga Bantu, Edmond Phuku Phuati, \\ Alain Lubo Musongela \\ Department of Physics, Faculty of Sciences, University of Kinshasa, Kinshasa, Democratic Republic of Congo \\ Email: albert.kazadi@unikin.ac.cd
}

How to cite this paper: Mutombo, R.M., Bantu, A.K.M., Phuati, E.P. and Musongela, A.L. (2017) Electromagnetic Waves Propagation Modes of Hot Plasma Located in Rotating Electromagnetic Field. Open Access Library Journal, 4: e3416.

https://doi.org/10.4236/oalib.1103416

Received: January 31, 2017

Accepted: March 27, 2017

Published: March 30, 2017

Copyright ( 2017 by authors and Open Access Library Inc.

This work is licensed under the Creative Commons Attribution International License (CC BY 4.0).

http://creativecommons.org/licenses/by/4.0/

\begin{abstract}
In this work it was established different electromagnetic wave propagation modes of hot plasma located in rotating electromagnetic field when the temperature is finite and when the temperature is considered as infinite. It is noticed that the plasma is completely transparent and isotropic with refractive index equal to unity when the temperature is infinite.
\end{abstract}

\section{Subject Areas}

Applied Physics

\section{Keywords}

Waves Propagation Modes, Hot Plasma, Rotating Electromagnetic Field

\section{Introduction}

Plasma Physics remains up today an area of great scientific interest. This is due to the multitude of applications that are involved in this area and also to the fact that none satisfactory explanation has not yet been given to several phenomena occurring in the plasma. The plasma does not yet so far reveal all its secrets [1].

In this work, we are going to establish the different electromagnetic wave propagation modes of hot plasma located in rotating electromagnetic field. To perform this, we have divided our study as follows.

In Section 2, we have presented the basis of the hot plasma dielectric tensor. In Section 3, we established the dispersion equation and the different propagation modes in the direction of the angle $\theta$ with the rotating magnetic field direction, in case of the finite temperatures and then in case that the temperature is growing to 
infinite value ending with discussion. Finally, the conclusion is in Section 4.

\section{Hot Plasma Dielectric Tensor [2]}

\subsection{Hypotheses}

The plasma, in study through these lines, is a gas completely ionized and obeys to the following double inequality:

$$
r_{0} \ll d_{e} \ll \lambda_{D}
$$

where parameters, $r_{0}, d_{e}$ and $\lambda_{D}$ are the Landau length, the average distance between particles and the Debye length.

This double inequality suggests that binary correlations are less important in plasma as well as collective interactions. This allows us to assimilate the studied plasma as an ideal gas [3].

As the plasma is hot, the aleatory component of the particle velocity and the temperature effects are taken into account.

From the above hypothesis, the plasma in examination can be described from Maxwell-Boltzmann distribution function as follows [4].

$$
f_{a}^{o}(p, t)=\left(2 \pi m_{a} k_{B} T_{a}\right)^{-3 / 2} \exp \left(-\frac{P^{2}}{2 m_{a} k_{B} T_{a}}\right)
$$

where $P_{a}=m_{a} v_{a}$ as the linear momentum of particle kind "a";

$k_{B}=$ Boltzmann constant;

$T_{a}=$ temperature of particle kind " $a$ " [1] [3].

\subsection{Dielectric Tensor [2] [5] [6]}

Examining this system in comparison with a referential rotating with the magnetic field and which axis $O Y$ coincides with the magnetic field vector $\boldsymbol{B}$ direction was the electrical conductivity tensor given by:

$$
\begin{aligned}
& \sigma_{i j}(\boldsymbol{k}, \Omega) \\
& =-\sum \frac{e_{a}^{2} n_{a}}{m_{a}} \int_{0}^{\infty} \mathrm{d} t \int \boldsymbol{p}_{a i}\left[1+\frac{(\boldsymbol{\omega} \wedge \boldsymbol{e})_{j}}{\Omega}\right]\left(\left(\frac{\partial f_{a}^{0}}{\partial \boldsymbol{p}}\right)_{i}\right)_{\boldsymbol{p} \rightarrow \boldsymbol{p}_{a}} \exp [i(\boldsymbol{k} \boldsymbol{r}+\Omega t)] \mathrm{d} \boldsymbol{p}
\end{aligned}
$$

where $e_{a}$ and $n_{a}$ are respectively the particles charge and number of kind " $a$ ".

This gives after integration which operating variable to change the electrical conductivity tensor whose elements are given below:

$$
\begin{gathered}
\sigma_{11}^{c}=\sigma_{33}^{c}=\frac{i \omega_{L} \Omega}{4 \pi\left(\Omega^{2}-\omega_{h}^{2}\right)}\left[1+\frac{(\omega \wedge e)_{1}}{\Omega}\right] \mathrm{e}^{-\frac{1}{2} m_{a} k_{B} T_{a} K_{a}^{2}} \\
\sigma_{13}^{c}=-\sigma_{31}^{c}=-\frac{\omega_{L}^{2} \omega_{h}}{4 \pi\left(\Omega^{2}-\omega_{h}^{2}\right)}\left[1+\frac{(\omega \wedge e)_{3}}{\Omega}\right] \mathrm{e}^{-\frac{1}{2} m_{a} k_{B} T_{a} K_{a}^{2}} \\
\sigma_{22}^{c}=\frac{i \omega_{L}^{2}}{4 \pi \Omega}\left[1+\frac{(\omega \wedge e)_{2}}{\Omega}\right] e^{-\frac{1}{2} m_{a} k_{B} T_{a} K_{a}^{2}} \\
\sigma_{12}^{c}=\sigma_{21}^{c}=\sigma_{23}^{c}=\sigma_{32}^{c}=0
\end{gathered}
$$


where $\omega_{h}$ is the rotating magnetic vector pulse, $\omega_{L}^{2}=\sum_{a} \frac{4 \pi e_{a}^{2} n_{a}}{m_{a}}$ is the Larmor frequency and the vector $\boldsymbol{K}_{a}$ is defined as below:

$$
\boldsymbol{K}_{a}(\boldsymbol{k}, t)=-\left\{\frac{(\boldsymbol{b} \cdot \boldsymbol{k}) \boldsymbol{b}}{m_{a}} t+\frac{(\boldsymbol{b} \Lambda \boldsymbol{k}) \Lambda \boldsymbol{b}}{m_{a} \omega_{h}} \sin \omega_{h} t+\frac{\boldsymbol{k} \Lambda \boldsymbol{b}}{m_{a} \omega_{h}}\left(1-\cos \omega_{h} t\right)\right\}
$$

where $\boldsymbol{b}$ is the unite vector along the magnetic field vector $\boldsymbol{B}$.

Finally as the dielectric tensor of our system is connected to the electrical conductivity tensor by:

$$
\varepsilon_{i j}=\delta_{i j}+i \frac{4 \pi}{\Omega} \sigma_{i j}^{c}
$$

Its elements will be:

$$
\begin{gathered}
\varepsilon_{1}=\varepsilon_{11}=\varepsilon_{33}=1-\eta_{1} \mathrm{e}^{-\frac{1}{2} m_{a} k_{B} T_{a} K_{a}^{2}} \\
i d=\varepsilon_{13}=\varepsilon_{31}^{*}=-i t \mathrm{e}^{-\frac{1}{2} m_{a} k_{B} T_{a} K_{a}^{2}} \\
\varepsilon_{2}=\varepsilon_{22}=1-\eta_{2} \mathrm{e}^{-\frac{1}{2} m_{a} k_{B} T_{a} K_{a}^{2}} \\
\varepsilon_{12}=\varepsilon_{21}=\varepsilon_{23}=\varepsilon_{32}=0
\end{gathered}
$$

where we admit that:

$$
\begin{gathered}
\eta_{1}=\frac{\omega_{L}^{2}}{\left(\Omega^{2}-\omega_{h}^{2}\right)}\left[1+\frac{(\omega \wedge e)_{1}}{\Omega}\right] \\
t=\frac{-\omega_{L}^{2} \omega_{h}}{\Omega\left(\Omega^{2}-\omega_{h}^{2}\right)}\left[1+\frac{(\omega \wedge e)_{3}}{\Omega}\right] \\
\eta_{2}=\frac{\omega_{L}^{2}}{\Omega^{2}}\left[1+\frac{(\omega \wedge e)_{2}}{\Omega}\right]
\end{gathered}
$$

The dielectric tensor of hot plasma located in a rotating electromagnetic field can be written as follow:

$$
\varepsilon_{i j}=\left(\begin{array}{ccc}
\varepsilon_{1} & 0 & -i d \\
0 & \varepsilon_{2} & 0 \\
i d & 0 & \varepsilon_{1}
\end{array}\right)
$$

or in two terms:

$$
\varepsilon_{i j}=\left(\begin{array}{lll}
1 & 0 & 0 \\
0 & 1 & 0 \\
0 & 0 & 1
\end{array}\right)-\left(\begin{array}{ccc}
\eta_{1} & 0 & -i t \\
0 & \eta_{2} & 0 \\
i t & 0 & \eta_{1}
\end{array}\right) \mathrm{e}^{-\frac{1}{2} m_{a} k_{B} T_{a} K_{a}^{2}}
$$

\section{Propagation Modes of Electromagnetic Waves}

We consider that the wave vector $\boldsymbol{k}$ makes a $\theta$ angle with the $O Y$ axis which carries the vector magnetic field $\boldsymbol{B}$ as shown in Figure 1.

The vector $\boldsymbol{k}$ in this case is given by:

$$
k=(k \sin \theta \sin \beta, k \cos \theta, k \sin \theta \cos \beta)
$$




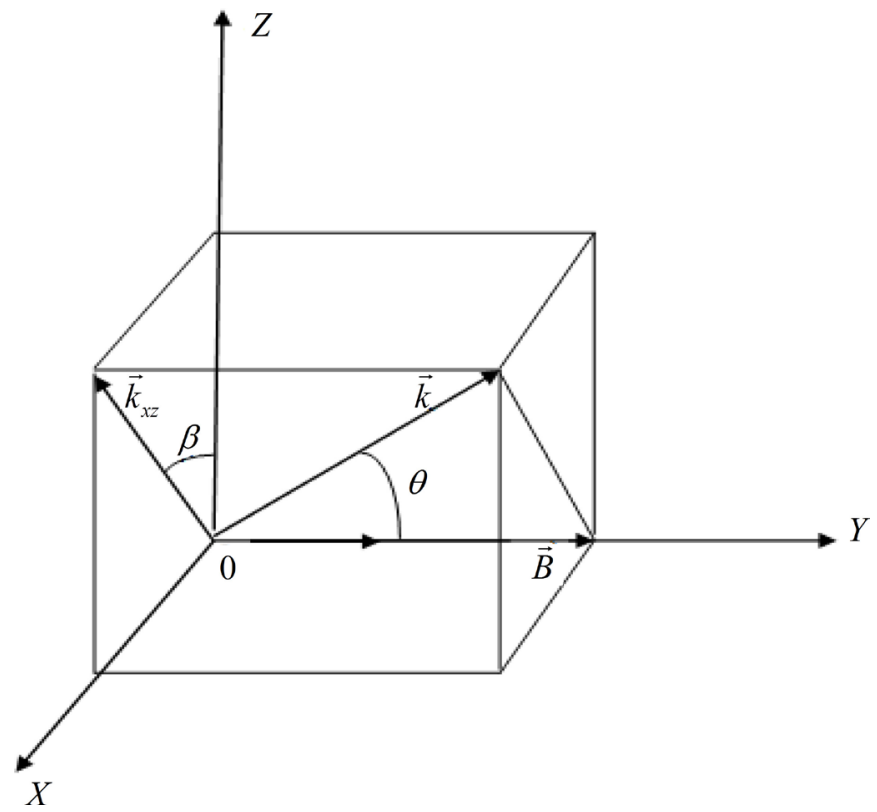

Figure 1. Representation of the $\boldsymbol{k}$ and $\boldsymbol{B}$ vectors.

And we have in this case: $\boldsymbol{k}_{x z}=\boldsymbol{k} \sin \theta$ is the projection of $\boldsymbol{k} \mathrm{d}$ in $X O Y$ plan 1) Dispersion equation [7]

From the following Maxwell equations:

$$
\begin{gathered}
\nabla_{r} \wedge \boldsymbol{B}(r, t)=\frac{1}{C} \frac{\partial \boldsymbol{E}(r, t)}{\partial t}+\frac{4 \pi}{C} \boldsymbol{J}^{t}(r, t) \\
\nabla_{r} \wedge \boldsymbol{E}(r, t)=-\frac{1}{C} \frac{\partial \boldsymbol{B}(r, t)}{\partial t}
\end{gathered}
$$

where $\boldsymbol{B}$ is the magnetic field, $\boldsymbol{E}$ the electric field and $\boldsymbol{J}^{t}$ the density of the induced electric current respectively.

When we replace in (2.3) $\quad \boldsymbol{B}$ by its expression derived from (2.2) we find:

$$
\nabla_{r}^{2} \boldsymbol{E}(r, t)-\nabla_{r}\left[\nabla_{r} \boldsymbol{E}(r, t)\right]=\frac{1}{C^{2}} \frac{\partial^{2} \boldsymbol{E}(r, t)}{\partial t^{2}}+\frac{4 \pi}{C^{2}} \frac{\partial \boldsymbol{J}^{i}(r, t)}{\partial t}
$$

By applying Fourier transformation to (2.4) we found:

$$
k^{2} \boldsymbol{E}(k, \Omega)-\boldsymbol{k}[\boldsymbol{k} \boldsymbol{E}(k, \Omega)]=\frac{\Omega^{2}}{C^{2}} \boldsymbol{E}(k, \Omega)+\frac{i 4 \pi \Omega}{C^{2}} \boldsymbol{J}^{\imath}(k, \Omega)
$$

And the $I^{\text {th }}$ component of the electric current density is given by:

$$
j_{i}^{i}(k, \Omega)=\sigma_{i j}(k, \Omega) E_{j}(k, \Omega)
$$

The equations' system above, will be written in terms of components as follow:

$$
\left(k_{i} k_{j}-\delta_{i j} k^{2}+\frac{\Omega^{2}}{C^{2}} \varepsilon_{i j}\right) E_{j}=0, i, j=1,2,3
$$

As the system (2.7) is linear and homogeneous it will admit a nontrivial solution only if the principal determinant of $(2.7)$ is zero [8], i.e.: 


$$
\left|k_{i} k_{j}-\delta_{i j} k^{2}+\frac{\Omega^{2}}{C^{2}} \varepsilon_{i j}\right|=0
$$

The relationship (2.8) is the dispersion equation of the electromagnetic wave in our plasma. Introducing (1.16) in Equation (2.8) while taking into account that the index of refraction " $n$ " of the wave in the medium is connected to the wave vector $\boldsymbol{k}$ and pulse wave " $\Omega$ " by the relation:

$$
n=\frac{k C}{\Omega}
$$

where " $\Omega$ " is pulse wave.

The determinant (2.8) in our case is given by:

$$
\left|\begin{array}{ccc}
n^{2} \sin ^{2} \theta \sin ^{2} \beta-n^{2}+\varepsilon_{1}^{c} & n^{2} \sin \theta \cos \theta \sin \beta & n^{2} \sin ^{2} \theta \sin \beta \cos \beta-i d \\
n^{2} \sin \theta \cos \theta \sin \beta & \varepsilon_{2}^{c}-n^{2} \sin ^{2} \theta & n^{2} \sin \theta \cos \theta \cos \beta \\
n^{2} \sin ^{2} \theta \sin \beta \cos \beta+i d & n^{2} \sin \theta \cos \theta \cos \beta & n^{2} \sin ^{2} \theta \cos ^{2} \beta-n^{2}+\varepsilon_{1}^{c}
\end{array}\right|=0
$$

After developing and removing any symmetric terms while combining other terms and taking into account the relationship:

$$
\cos ^{2} \beta+\sin ^{2} \beta=1
$$

The expression (2.10) is reduced to:

$$
\begin{aligned}
\Delta= & \left(\varepsilon_{1}^{c} \sin ^{2} \theta+\varepsilon_{2}^{c} \cos ^{2} \theta\right) n^{4}+\left[\left(d^{2}-\varepsilon_{1}^{c 2}\right) \sin ^{2} \theta-\varepsilon_{1}^{c} \varepsilon_{2}^{c}\left(1+\cos ^{2}\right)\right] n^{2} \\
& +\left(\varepsilon_{1}^{c 2} \varepsilon_{2}^{c 2}-d^{2} \varepsilon_{2}^{c}\right)=0
\end{aligned}
$$

Finally when considering

$$
D=\varepsilon_{1}^{c}+d, G=\varepsilon_{1}^{c}-d
$$

Putting (2.13) in (2.12) we have:

$$
A n^{4}-B n^{2}+C=0
$$

This expression (2.14) is the general dispersion relation of hot plasma located in rotating electromagnetic field, where coefficients $A, B$ and $C$ are given by:

$$
\left\{\begin{array}{l}
A=\varepsilon_{1}^{c} \sin ^{2} \theta+\varepsilon_{2}^{c} \cos ^{2} \theta \\
B=D G \sin ^{2} \theta-\varepsilon_{1}^{c} \varepsilon_{2}^{c}\left(1+\cos ^{2} \theta\right) \\
C=\varepsilon_{2}^{c} D G
\end{array}\right.
$$

When solving Equation (2.14) with respect to the square of the refractive index " $n 7^{2 "}$ we get:

$$
n^{2}=\frac{B \pm \sqrt{B^{2}-4 A C}}{2 A}
$$

However, it is more interesting to solve (2.12) with respect to the angle $\theta$. Indeed by dividing the terms of (2.12) by $\cos ^{2} \theta$ while taking account of:

$$
\left\{\begin{array}{l}
D=\varepsilon_{1}+d \\
G=\varepsilon_{1}-d \\
\frac{1}{\cos ^{2} \theta}=1+\operatorname{tg}^{2} \theta
\end{array}\right.
$$


We got:

$$
\operatorname{tg}^{2} \theta=\frac{-\varepsilon_{2}\left(n^{2}-D\right)\left(n^{2}-G\right)}{\left(\varepsilon_{1} n^{2}-D G\right)\left(n^{2}-\varepsilon_{2}\right)}
$$

As we observe, the angle $\beta$ does not appear in the relation (2.14) nor in (2.18). Only the angle " $\theta$ " is the angle between the vector wave $\boldsymbol{k}$ and the vector magnetic field $\boldsymbol{B}$ found in the two relationships (2.14) and (2.18). This suggests that not only the electromagnetic wave propagation modes depend only on the angle between the vectors $\boldsymbol{k}$ and $\boldsymbol{B}$ but also that all directions of revolution generated by vector $\boldsymbol{k}$ making an angle $\theta$ with $\boldsymbol{B}$, around the axis $O Y$ carrying the vector $\boldsymbol{B}$, are equivalent.

With this reality, we can use a simpler case of an oblique vector to have an illustration of the different propagation modes in the event the wave vector $\boldsymbol{k}$ make any angle $\Theta$ with the vector magnetic field $\boldsymbol{B}$.

\section{2) Propagation modes of electromagnetic waves}

In case $\beta$ is the angle right $(\beta=\pi / 2)$.

This corresponds to a situation where the vector $\boldsymbol{k}$ is situated in the $X O Y$ plan as shown in Figure 2.

In this case the vector $\boldsymbol{k}$ is given by:

$$
k=(k \sin \theta, k \cos \theta, 0)
$$

and the system of Equation (2.7) is given:

$$
\left(\begin{array}{ccc}
\varepsilon_{1}^{c}-n^{2} \cos ^{2} \theta & n^{2} \cos \theta \sin \theta & -i d \\
n^{2} \cos \theta \sin \theta & \varepsilon_{2}^{c}-n^{2} \sin ^{2} \theta & 0 \\
i d & 0 & \varepsilon_{1}^{c}-n^{2}
\end{array}\right)\left(\begin{array}{l}
E_{x} \\
E_{y} \\
E_{z}
\end{array}\right)=0
$$

We can note that in this system, three wave propagation modes each represented by a line of the equation system (2.20). For ease of calculation, consider these modes starting from the third line to the first

a) First Mode: third line

$$
i d E_{x}+0 E_{y}+\left(\varepsilon_{1}^{c}-n^{2}\right) E_{z}=0
$$

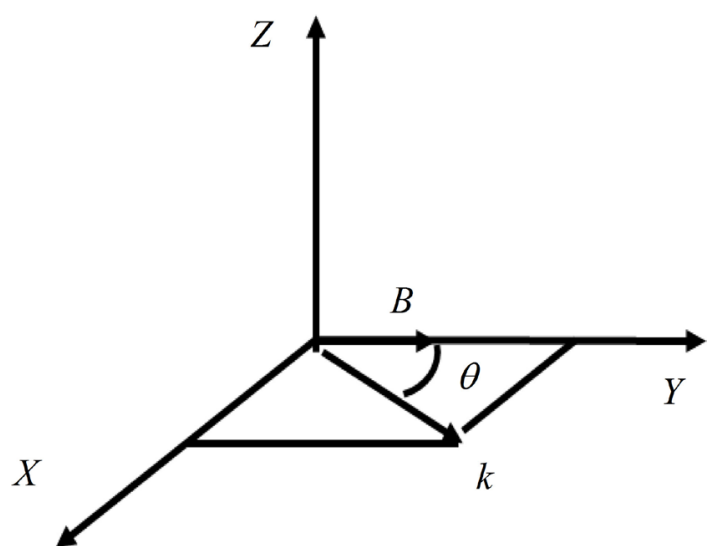

Figure 2. The vector $\boldsymbol{k}$ making an angle $\Theta$ with the vector $\boldsymbol{B}$ carried by the $O Y$ axis lies in the $X O Y$ plane. 
From the Equation (2.21) we can write $E_{z}$ relative to $E_{x}$ as follows:

$$
E_{z}=-\frac{i d}{\varepsilon_{1}^{c}-n^{2}} E_{x}
$$

The wave represented by the third line is illustrated by Figure 3 [9]

In this figure, we can see that the electric field vector $E_{x}$ is the vector sum of two components $E_{l}$ and $E_{t}$ such as:

$E_{l}$ is oriented in the direction of the vector $\boldsymbol{k}$ and is the longitudinal component of the wave. It is given by:

$$
E_{l}=E_{x} \sin \theta
$$

while $E_{t}$ is oriented along the direction perpendicular to the wave vector $\boldsymbol{k}$ in the $X O Y$ plane and is expressed as following:

$$
E_{t}=E_{x} \cos \theta
$$

Therefore, the transverse part of the wave consists of two components respectively oriented as follows:

The first, in the direction perpendicular to $\boldsymbol{k}$ in plane XOY given by Equation (2.24); and the second in the direction of $O Z$, as expressed by Equation (2.22).

$$
\boldsymbol{E}_{T}=\boldsymbol{E}_{z}+\boldsymbol{E}_{t}
$$

With $\boldsymbol{E}_{T}$ the transverse component of the wave

\section{Polarization}

The transverse component of the wave is elliptically polarized in the plane perpendicular to the vector $\boldsymbol{k}$.

Indeed we have:

$$
\frac{i E_{t}}{E_{z}}=-\frac{\varepsilon_{1}^{c}-n^{2}}{d} \cos \theta \neq 1
$$

So we have a hybrid mode whose transverse component is elliptically polarized in the plane perpendicular to the wave vector $\boldsymbol{k}$.

\section{b) Second mode: the second line}

The second wave, shown in Figure 4, is given by the second line of our equation system (2.20) as set out below:

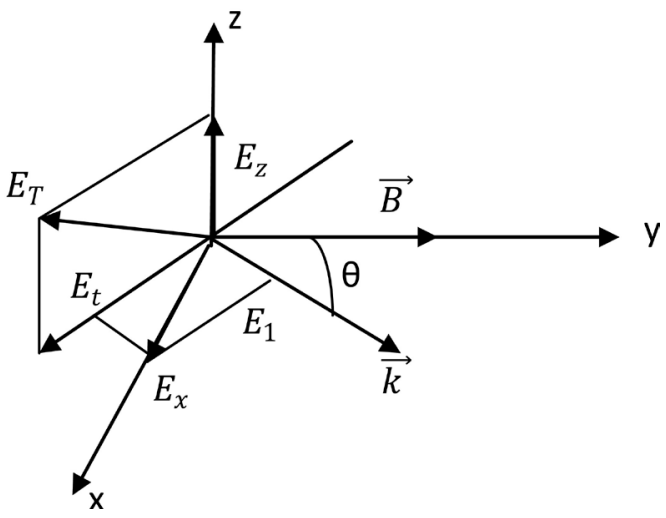

Figure 3. The component $E_{x}$ is the result of two vectors $E_{l}$ and $E_{t}$. 


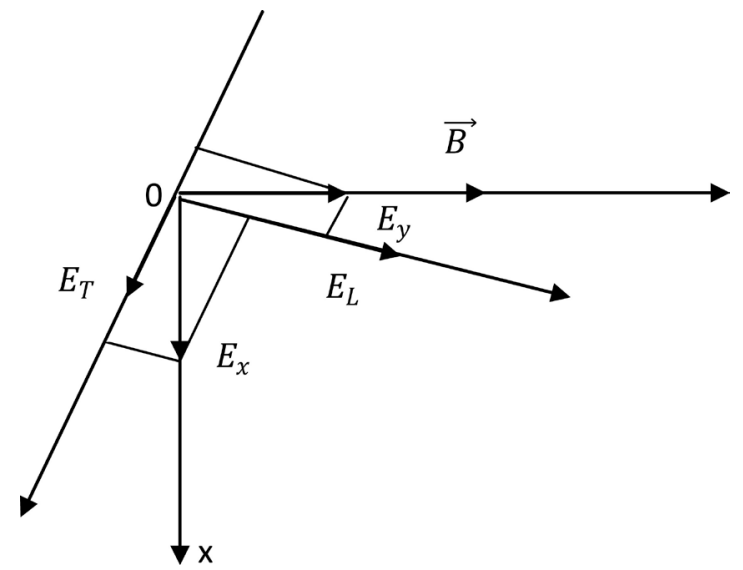

Figure 4. Representation of a plane wave propagating in the $X O Y$ plan.

$$
n^{2} \cos \theta \sin \theta E_{x}+\left(\varepsilon_{2}^{c}-n^{2} \sin ^{2} \theta\right) E_{y}+0 E_{z}=0
$$

From this Equation (2.27) we can express $E_{y}$ versus $E_{x}$

$$
E_{y}=-\frac{n^{2} \cos \theta \sin \theta}{\varepsilon_{2}^{c}-n^{2} \sin ^{2} \theta} E_{x}
$$

This wave as shown in Figure 4, has two components:

(a) A longitudinal component given by:

$$
E_{L}^{\prime}=E_{x} \sin \theta+E_{y} \cos \theta
$$

(b) A transverse component as:

$$
E_{T}^{\prime}=E_{x} \cos \theta-E_{y} \sin \theta
$$

Taking into account (2.28) we have:

$$
E_{L}^{\prime}=E_{x} \sin \theta-E_{x} \frac{n^{2} \cos \theta \sin \theta}{\varepsilon_{2}^{c}-n^{2} \sin ^{2} \theta} \cos \theta
$$

After development we have:

$$
E_{L}^{\prime}=E_{x} \frac{\left(\varepsilon_{2}^{c}-n^{2}\right) \sin \theta}{\varepsilon_{2}^{c}-n^{2} \sin ^{2} \theta}
$$

This is the longitudinal component.

And the transverse component is expressed by:

$$
E_{L}^{\prime}=E_{x} \cos \theta+E_{x} \frac{n^{2} \cos \theta \sin \theta}{\varepsilon_{2}^{c}-n^{2} \sin ^{2} \theta} \sin \theta .
$$

Finally:

$$
E_{L}^{\prime}=E_{x} \frac{\varepsilon_{2}^{c} \cos \theta}{\varepsilon_{2}^{c}-n^{2} \sin ^{2} \theta}
$$

Polarisation

$$
\frac{E_{L}^{\prime}}{E_{T}^{\prime}}=\frac{\left(\varepsilon_{2}^{c}-n^{2}\right) \sin \theta}{\varepsilon_{2}^{c} \cos \theta}=\frac{\varepsilon_{2}^{c}-n^{2}}{\varepsilon_{2}^{c}} \operatorname{tg} \theta \neq 1
$$

We have a hybrid wave propagation elliptically polarized in the $X O Y$ plan. 


\section{c) Third mode: the first line}

The third mode represented by the first equation of the system (2.20) is given by the following expression:

$$
\left(\varepsilon_{1}^{c}-n^{2} \cos ^{2} \theta\right) E_{x}+n^{2} \cos \theta \sin \theta E_{y}-i d E_{z}=0
$$

This mode has:

- a longitudinal component given by the $E_{L}^{\prime}$ of the second embodiment such as it is expressed by Equation (2.32).

- while the transverse portion, in addition to the component $E_{T}^{\prime}$ given by the relation (2.33) and oriented along the perpendicular to the wave vector $\boldsymbol{k}$ in the XOY plane, it has a further component in the direction $O Z$ as given by the relation (2.22) and denoted $E_{z}$.

The transverse portion in this mode is elliptically polarized in the plane perpendicular to the wave vector $\boldsymbol{k}$.

Indeed we have:

$$
\frac{i E_{T}^{\prime}}{E_{z}}=-\frac{\left(\varepsilon_{1}^{c}-n^{2}\right) \varepsilon_{2}^{c} \cos \theta}{\mathrm{d}\left(\varepsilon_{2}^{c}-n^{2} \sin ^{2} \theta\right)} \neq 1
$$

We have three hybrid modes of which one is elliptically polarized in the propagation plane and the others two transverse components are elliptically polarized in the plane perpendicular to the direction of propagation.

\section{3) Temperature $t_{a}$ tend to infinity}

The relation (1.18) giving hot plasma dielectric tensor includes two terms of which the second would vanish when the $T_{a}$ tends to infinity. The plasma dielectric tensor is reduced in this case to unit matrix of order 3 given below:

$$
\varepsilon_{i j}^{c}=\delta_{i j}=\left(\begin{array}{ccc}
1 & 0 & 0 \\
0 & 1 & 0 \\
0 & 0 & 1
\end{array}\right)
$$

Taking into account (2.19), the system of Equation (2.7) can be written:

$$
\left(\begin{array}{ccc}
1-n^{2} \cos ^{2} \theta & n^{2} \sin \theta \cos \theta & 0 \\
n^{2} \sin \theta \cos \theta & 1-n^{2} \sin ^{2} \theta & 0 \\
0 & 0 & 1-n^{2}
\end{array}\right)\left(\begin{array}{l}
E_{x} \\
E_{y} \\
E_{z}
\end{array}\right)=0
$$

The main determinant of (2.38) is given by:

$$
\Delta=\left(1-n^{2}\right)\left[\left(1-n^{2} \cos ^{2} \theta\right)\left(1-n^{2} \sin ^{2} \theta\right)-n^{4} \sin ^{2} \theta \cos ^{2} \theta\right]
$$

After developing the terms in bracket of Equation (2.39) and regrouping the same terms, we finally have:

$$
\Delta=\left(1-n^{2}\right)^{2}
$$

For the same reason mentioned in the previous case, namely that the system (2.38) can admit a nontrivial solution only if the principal determinant of the system is zero, we will have:

$$
\left(1-n^{2}\right)=0
$$


The relation (2.41) is the dispersion relation for the hot plasma located in an electromagnetic field during rotation, when the temperature $T_{a}$ of the particles becomes extremely high.

We see that as the temperature approaches infinite values, the propagation modes are the same in all directions and not dependent over the direction of $\boldsymbol{k}$.

From the dispersion relation (2.41) we see that the refraction index " $n$ " of transverse waves is equal to unity independently of the direction of wave propagation. This also means that the phase velocity is equal to the light speed in vacuum. Taking the case where the wave vector $\boldsymbol{k}$ is parallel to the axis $O X$.

The angle $\theta$ is then equal to a right angle $(\theta=\pi / 2)$. The system (2.38) is then written:

$$
\left(\begin{array}{ccc}
1 & 0 & 0 \\
0 & 1-n^{2} & 0 \\
0 & 0 & 1-n^{2}
\end{array}\right)\left(\begin{array}{l}
E_{x} \\
E_{y} \\
E_{z}
\end{array}\right)=0
$$

The system (2.42) shows the existence of three waves:

1) a longitudinal wave with the electric field oriented in the direction of propagation $O X$.

2) two identical transverse waves with electric field linearly polarized respectively on the $O Y$ axis and the $O Z$ axis.

This situation can be explained by the fact that the thermal motion of the particles becomes so intense that the effects of the magnetic field become negligible.

\section{Conclusions}

We have derived the expression of the dielectric tensor of hot plasma located in a rotating electromagnetic field. We noticed that the temperature effects are expressed by an exponential factor depending on the temperature. As the temperature is not too high, we found three modes of elliptical hybrid polarization such that one is polarized in the wave propagation plane while two others have their transverse components elliptically polarized in the plane perpendicular to the direction of the vector $\boldsymbol{k}$.

The presence of the angel $\theta$ in the dispersion relation shows the anisotropic character of our medium in study. When the temperature $T_{a}$ tends to be extremely high, our medium in study not only appears isotropic, but also has a refractive index equal to unity. In any direction of propagation we have a longitudinal mode and two ordinary transverse waves linearly polarized with the same phase velocity equal to the speed of light in vacuum $\left(V_{p h}=C\right)$.

\section{Acknowledgements}

The financial support from the Belgium Cooperation CUD through the project KIN04 is gratefully acknowledged.

\section{References}

[1] Delcroix, J.L. and Bers, A. (1994) Physique des plasmas, Savoirs actuels, CNRS 
EDITION.

[2] Kazadi Mukenga Bantu, A., et al. (2009) Tenseur diélectrique du plasma chaud dans un champ électromagnétique en rotation, physica scripta. Volume 80.

[3] Klimontovich, Y.L. (1967) The Statistical Theory of Non-Equilibrium Processus in Plasma. Pergamon Press Ltd., London.

[4] Pierrard, V. (2009) Environnement Spatial de la terre. U.C.L., Presses Universitaires de Louvain.

[5] Akhiezer, A. and Peletminski, S. (1980) Les méthodes de la physique statistique. Mir, Moscou.

[6] Lofo, L.B. and Kazadi, M.B. (1996) Sur l'expression du tenseur diélectrique du plasma dans un champ électromagnétique en rotation, phisica sripta. Volume 54.

[7] Kazadi Mukenga Bantu, A., et al. (2016) Dispersion Relation of Waves in Hot Plasma Located in Rotating Electromagnetic Field. American Journal of Physics and Applications, 4, 1-4. https://doi.org/10.11648/j.ajpa.20160401.11

[8] Kuroch, A. (1973) Cours d'Algèbre supérieure. MIR, Moscou.

[9] Parks, G.K. (2004) Physics of Space Plasma, West View.

Submit or recommend next manuscript to OALib Journal and we will provide best service for you:

- Publication frequency: Monthly

- 9 subject areas of science, technology and medicine

- Fair and rigorous peer-review system

- Fast publication process

- Article promotion in various social networking sites (LinkedIn, Facebook, Twitter, etc.)

- Maximum dissemination of your research work

Submit Your Paper Online: Click Here to Submit

Or Contact service@oalib.com 